\title{
DECOMPOSITIONS OF MEASURE ALGEBRAS AND SPACES
}

\author{
BY \\ DOROTHY MAHARAM
}

\section{Introduction.}

1.1. Let $(\Omega, B, \mu)$ be a measure space, in which the measure $\mu$ is $\sigma$-infinite $\left.{ }^{1}\right)$, and let $(E, \mu)$ be the associated measure algebra of measurable sets (B) modulo null sets. If a field $\mathcal{A}$ of measurable sets is given in $\Omega$, corresponding to a sub-algebra $A$ of $E$, it is known (see $[4,1,3]$ ) that, under suitable hypotheses $\left({ }^{2}\right), \Omega$ can be expressed as a direct sum of measure spaces $Y_{x}$ over a base-space $X$ whose measurable sets correspond, in a simple way, to those of $A$. In particular, if $A$ is the field of sets which are invariant under a measure-preserving transformation (or a flow) on $\Omega$, the resulting decomposition gives a decomposition of the transformation (or flow) into ergodic parts. The object of the present paper is to extend and sharpen these results, by showing that these direct sum decompositions can (roughly speaking) be imbedded in a direct product $X \times Y$ of measure spaces, the spaces $Y_{x}$ becoming merely the sections $x=$ constant of a suitable measurable subset of this product. The significance of this sharpening consists essentially in that a direct sum of measure spaces $Y_{x}$ over a base-space $X$ is not completely determined when the measure spaces $Y_{x}$ and $X$ are known, since the field of measurable sets is largely undetermined, whereas of course a direct product $X \times Y$ is so determined by the factors. From the present point of view, the fact that $\mu(\Omega)$ may be infinite constitutes a further nontrivial generalization, as $\mu$ may well take only the values 0 and $\infty$ for sets in $A$.

We consider first the "algebraic" problem of decomposing $E$ with respect to $A$; this is solved $(\$ 3)$, after some preliminary remarks on product spaces (\$2), by introducing an abstract-valued measure (or, more precisely, a corresponding equivalence relation) into $E$, and appealing to the analysis of abstract-valued measure algebras given in [8]. Other algebraic decomposition and imbedding theorems, due to Nikodým, Gleason, and Dieudonné, are then deduced (\$4), in somewhat sharpened form, and without the assumption of finite total measure. The case in which $A$ arises as the set of elements invariant under a group of measure-preserving transformations is then char-

Presented to the Society, April 29, 1950; received by the editors November 1, 1949.

(1) That is, $\mu$ is a non-negative countably additive set-function on the field $B$ of subsets of $\Omega$, and $\Omega=\bigcup B_{n}, B_{n} \in B$, where $\mu\left(B_{n}\right)<\infty$. Contrary to the usual practice, we do not require that $\mu(\Omega)$ be finite.

(2) Notably "normality"; see [5] and [3, p. 41]. This hypothesis was inadvertently omitted in [1] and [4]. Numbers in brackets refer to the bibliography at the end of the paper. 
acterized (\$5). We finally deduce the analogous "point" theorems for normal measure spaces, incidentally showing (Theorem $5,6.5$ ) that, under reasonable hypotheses, an arbitrary direct sum is in fact (roughly speaking) imbeddable in a direct product. The resulting decomposition of a measure-preserving transformation into ergodic parts (Theorem 6, 7.2) is an amplification of a remark in [8, p. 329], and gives a fairly complete analysis of the relation between these ergodic parts and the whole transformation.

1.2. Notations. As far as possible, we follow the notations of [8]; the necessary departures will be obvious. All measure spaces considered will be assumed to have $\sigma$-finite (but not necessarily finite) measures. Similarly, the term "measure algebra" $(E, \mu)$ will mean a Boolean $\sigma$-algebra on which $\mu$ is a countably additive, non-negative measure which is $\sigma$-finite and positive (or "reduced"-that is, $\mu(x)=0$ if and only if $x=0$ ). There is usually no loss of generality in assuming that the measure spaces considered are complete (that is, that every subset of a null set is measurable), and this assumption will be made unless the contrary is indicated. A point-isometry, or isometry, between two measure spaces, is a 1-1 mapping of one onto the other which preserves measurability and measure (both ways). The definition of "isometry" for measure algebras is obvious, and an isometry between the algebras associated with two measure spaces will be called a set-isometry, or set-mapping, of the spaces. If $R$ is the representation space of an algebra $E$, we use $x^{*}$ to denote the open-closed subset of $R$ which corresponds to $x \in E$; it may happen that several representation spaces (of various sub-algebras of $E$ ) are involved, but the space to which $x^{*}$ refers will be clear from the context.

\section{Realizations and direct products.}

2.1. The transition from the abstract-valued measures, which will be the principal algebraic tool in what follows, to ordinary numerical measures, will be obtained by comparing two kinds of direct products of measure algebras; thus we begin with some fairly obvious remarks about these products.

If $(E, \mu)$ is the measure algebra associated with a measure space $(\Omega, \mu)$, we call $(\Omega, \mu)$ a realization of $(E, \mu)$. Every $(E, \mu)$ has at least one realization, obtained by taking $\Omega=$ representation space $R$ of $E$, the measurable sets forming the field generated by the open-closed subsets of $R$.

2.2. Any two measure-spaces $\left(\Omega_{1}, \mu_{1}\right),\left(\Omega_{2}, \mu_{2}\right)$, have a direct product, defined in a well known way (cf. [11, pp. 82-]). The measure algebra $(E, \mu)$ associated with this product is defined to be the direct product of the algebras $\left(E_{1}, \mu_{1}\right),\left(E_{2}, \mu_{2}\right)$ associated with the factor spaces. The product $(E, \mu)$ is determined uniquely (to within isometry) by the factors $\left(E_{1}, \mu_{1}\right)$ and $\left(E_{2}, \mu_{2}\right)$, and is independent of the choice of their realizations; this follows from the fact that $(E, \mu)$ is characterized by the following properties: (i) it is a measure algebra containing sub-algebras $E_{1}^{\prime}, E_{2}^{\prime}$ which are algebraically isomorphic to $E_{1}, E_{2}$ respectively, (ii) $E$ is generated by $E_{1}^{\prime} \cup E_{2}^{\prime}$, and (iii) if $x_{i}^{\prime} \in E_{i}^{\prime}$ corresponds to $x_{i} \in E_{i}(i=1,2)$, then $\mu\left(x_{1}^{\prime} x_{2}^{\prime}\right)=\mu_{1}\left(x_{1}\right) \mu_{2}\left(x_{2}\right)$ (with the conven- 
tion $\infty \cdot 0=0=0 \cdot \infty$ ). Further, the product measure will be $\sigma$-finite (assuming, of course, $\sigma$-finiteness on the factors).

2.3. Similarly, the "abstract product" $\left(\Omega_{1}, \mu_{1}\right) \times \Omega_{2}$ of two measure spaces can be defined as follows. The measurable sets $H$ are those of $\left(\Omega_{1}, \mu_{1}\right) \times\left(\Omega_{2}, \mu_{2}\right)$, but the "measure" $M\{H\}$ is the non-negative measurable function on $\Omega_{2}$ whose value at $p \in \Omega_{2}$ is $\mu_{1}\{q \mid(q \times p) \in H\}$, modulo functions on $\Omega_{2}$ which vanish outside null sets. This "measure" is countably additive in the obvious sense, and is $\sigma$-finite since $\mu_{1}$ is so. The "abstract product" $\left(E_{1}, \mu_{1}\right) \times E_{2}$ of the associated algebras is defined to be the abstract-valued measure algebra associated with $\left(\Omega_{1}, \mu_{1}\right) \times \Omega_{2}$; again it is independent of the choice of realizations of $\left(E_{1}, \mu_{1}\right)$ and $E_{2}$, as follows from the characterization given in [8, p. 284] $\left.{ }^{(3}\right)$.

2.4. The relation between the abstract and numerical products is now obvious from Fubini's theorem: the numerical direct product $\left(E_{1}, \mu_{1}\right) \times\left(E_{2}, \mu_{2}\right)$ is algebraically isomorphic to the abstract direct product $\left(E_{1}, \mu_{1}\right) \times E_{2}$, and its measure is given by: $\mu(H)=\int M\{H\} d \mu_{2}$.

3. Decomposition with respect to a sub-algebra.

3.1. Let $A$ be a given $\sigma$-sub-algebra of a measure algebra $(E, \mu) ; \mu$ need not necessarily be $\sigma$-finite on $A\left({ }^{4}\right)$. We shall show that, roughly speaking, $E$ can be decomposed into a countable number $\left(^{5}\right)$ of pieces, each of which is the direct product of some measure algebra with an ideal in $A$ (with a new measure). The precise results are stated below $(3.8,4.1)$. The first step is to dispose of the elements of $E$ which are of order 0 over $A\left(^{6}\right)$. We recall that $x(\in E)$ is of order 0 (the phrase "over $A$ " will generally be omitted) if and only if the ideal $E(x)$ (of elements $y \in E$ satisfying $y \leqq x$ ) coincides with $x A$, the set of elements of the form $x a, a \in A$. Thus $o$ is of order 0 , and if $y \leqq x$ and $x$ is of order 0 , then so is $y$.

Let $g_{1}, g_{2}, \cdots$ be a maximal disjoint collection (necessarily countable $(5)$ ) of nonzero elements of order 0 , and write $e_{0}=\bigvee g_{n}, e_{*}=e-e_{0}$. Thus $E\left(e_{*}\right)$ contains no nonzero element of order 0 .

3.2. Given $x \in E$, the smallest element of $A$ satisfying $a \geqq x$ will be called the closure of $x$, written $\bar{x}$ or $\mathrm{Cl}(x)$. We note that

(1) $\mathrm{Cl}\left(\mathrm{V} x_{n}\right)=\mathrm{V}\left(\mathrm{Cl}\left(x_{n}\right)\right)$.

(2) $\mathrm{Cl}(a x)=a \bar{x}$, if $a \in A$.

(3) If $x$ is of order 0 , and $y \leqq x$, then $x \bar{y}=y$, and the ideals $E(x), A(\bar{x})$ are isomorphic under the correspondence $y \rightarrow a=\bar{y}, a \rightarrow y=x a$.

(3) Compare [8, pp. 283-290]. In [8] the representation space realizations were used; this has the advantage of enabling the function $M\{H\}$ to be chosen uniquely (as a continuous function).

(4) That is, there need not exist elements $a_{n} \in A$ such that $\bigvee a_{n}=e$ and $\mu\left(a_{n}\right)<\infty$.

(5) Throughout this paper, "countable" means "at most countable," and "sequences" may terminate finitely except where the contrary is explicitly stated.

(6) See $[8$, p. 301]. The elements of order 0 over $A$ have properties similar to some of the properties of the "indecomposable" elements in [8, pp. 295, 296]; in fact, the indecomposable elements in [8] are of order 0 over the invariant algebra. 
To prove (3), we note that, since $x$ is of order $0, y=a x$ where $a \in A$; hence, from (2), $x \bar{y}=x a \bar{x}=a x=y$, and the result follows.

(4) If $x_{1}, x_{2}, \cdots$ are elements of order 0 having disjoint closures, then $\mathrm{V} x_{n}$ is of order 0 . (Cf. [8, 9.3].)

For if $y \leqq \mathrm{~V} x_{n}$, we have $y x_{n}=a_{n} x_{n}$ where $a_{n} \in A$ and we can assume $a_{n} \leqq \mathrm{Cl}\left(x_{n}\right)$. Then $y=\mathrm{V} y x_{n}=\left(\mathrm{V} a_{n}\right)\left(\mathrm{V} x_{n}\right)=a x$, where $a \in A$.

(5) There exist disjoint elements $d_{1}, d_{2}, \cdots$, each of order 0 , such that (i) $e_{0}=\bigvee d_{n}$, (ii) $\bar{e}_{0}=\bar{d}_{1} \geqq \bar{d}_{2} \geqq \cdots$.

Proof. By construction, $e_{0}=\bigvee g_{n}$, where the elements $g_{n}$ are disjoint and of order 0 . The elements $g_{n}-\mathrm{Cl}\left(g_{1} \bigvee g_{2} \bigvee \ldots \vee g_{n-1}\right), n=1,2, \cdots$, have disjoint invariant closures; hence their supremum, $d_{1}$ say, is of order 0 (from (4)). Further, $g_{1} \leqq d_{1} \leqq \bigvee g_{n}=e_{0}$, and $\bar{d}_{1}=\mathrm{V}\left[\mathrm{Cl}\left(g_{n}\right)-\left\{\mathrm{Cl}\left(g_{1}\right) \vee \cdots \vee \mathrm{Cl}\left(g_{n-1}\right)\right\}\right]$ $=\mathrm{Cl}\left(\mathrm{V}_{n}\right)=\bar{e}_{0}$.

When $d_{k}$ has been defined, we write $g_{n, k}=g_{n}-\left(d_{1} \bigvee \cdots \bigvee d_{k}\right)$, and define $d_{k+1}=\mathrm{V}_{n \geqq k+1}\left\{g_{n, k}-\mathrm{Cl}\left(g_{k+1, k} \bigvee \ldots \vee g_{n-1, k}\right)\right\}$. As before, $d_{k+1}$ is of order 0 , $g_{n, k} \leqq d_{k+1} \leqq e_{0}-\left(d_{1} \bigvee \cdots \bigvee d_{k}\right)$, and $\mathrm{Cl}\left(d_{k+1}\right)=\mathrm{Cl}\left(e_{0}-\left(d_{1} \bigvee \cdots \vee d_{k}\right)\right)$. It is easy to see that the desired properties follow.

Now define a measure $\mu_{n}$ on the ideal of $\bar{d}_{n}$ in $A$ by:

$$
\mu_{n}(a)=\mu\left(a d_{n}\right) \quad\left(a \in A, a \leqq \bar{d}_{n}\right) .
$$

Clearly $\mu_{n}$ is a $\sigma$-additive and $\sigma$-finite measure on $A\left(\bar{d}_{n}\right)$, and further (from (2)) $\mu_{n}$ is positive. From (3) we have at once:

(7) The ideal $\left(E\left(d_{n}\right), \mu\right)$ is isometric to the ideal $\left(A\left(\bar{d}_{n}\right), \mu_{n}\right)$.

3.3. Now consider the ideal $E\left(e_{*}\right)$. Write

(1) $x \sim y$ if and only if $\mu(a x)=\mu(a y)$ for all $a \in A\left(^{7}\right)$. Obviously this is an equivalence relation on $E\left(e_{*}\right)$. We shall prove:

TheOREM 1. $\left(E\left(e_{*}\right), \sim\right)$ satisfies the postulates of $[8$, p. 281$]$; further, it has no nonzero indecomposable elements [8, p. 295], and its invariant elements $\left[8\right.$, p. 290] form precisely the relative algebra $e_{*} A\left({ }^{8}\right)$.

The proof requires several lemmas. To simplify the notation, we shall drop the asterisks in 3.4-3.7, referring to $e_{*}, E\left(e_{*}\right), e_{*} A$ simply as $e, E, A$.

3.4. Lemma 1. Let $g, h$ be two $\sigma$-additive finite numerical functions on $a$ $\sigma$-algebra $B$ satisfying the countable chain condition. There exists $b_{0} \in B$ such that (i) if $b \in B\left(b_{0}\right), g(b) \leqq h(b)$, (ii) if $b \in B\left(-b_{0}\right), g(b)>h(b)$ unless $b=o$.

This is a form of the Jordan decomposition theorem. To prove it, an easy "exhaustion" argument gives the existence of a maximal element $b^{\prime} \in B$ such

( $\left.{ }^{7}\right)$ If $\mu$ is $\sigma$-finite on $A$, this is equivalent to defining an abstract-valued measure on $E\left(e_{*}\right)$ by: $M\{x\}=$ Radon-Nikodým function for the additive set-function $\mu(a x)$ on a realization of $(A, \mu)$, and writing $x \sim y$ to mean $M\{x\}=M\{y\}$ p.p.

(8) Consequently no confusion can arise from the two definitions of "closure" (3.2 above and $[8$, p. 291]), as these coincide. 
that, whenever $b \in B\left(b^{\prime}\right)$ and $b \neq 0$, then $g(b)-h(b)>0$. We have only to take $b_{0}=-b^{\prime}$.

Lemma 2. Given $x \in E$, and given a $\sigma$-additive non-negative numerical function $f$ on $A$, not identically 0 , such that $0 \leqq f(a) \leqq \mu(x a)$ for all $a \in A$, there exists $y \leqq x$ such that $y \neq 0$ and $\mu(y a) \leqq f(a)$ for all $a \in A$.

First assume $\mu(x)<\infty$. Now apply Lemma 1 to the algebra $A(\bar{x})$ and the functions $f(a)$ and $\epsilon \mu(x a)$, where $\epsilon>0$. If the resulting element $b_{0}$ were $o$ for all $\epsilon>0, f$ would vanish identically, contrary to hypothesis. Hence there exist $\epsilon>0$ and $b_{0} \in A(\bar{x})$, with $b_{0} \neq 0$, such that

$$
f(a) \geqq \epsilon \mu(x a)
$$

for all $a \in A\left(b_{0}\right)$.

We shall show that there exists $y_{1} \leqq x b_{0}$ such that $y_{1} \neq 0$ and

$$
\mu\left(y_{1} a\right) \leqq \mu(x a) / 2 \quad \text { for all } a \in A\left(\bar{y}_{1}\right) .
$$

For $x b_{0} \neq 0$, since otherwise (from 3.2(2)) $\bar{x} b_{0}=o$, contradicting $b_{0} \neq 0$. Hence $x b_{0}$ is not of order 0 over $A$; that is, there exists $y \leqq x b_{0}$ such that $y \notin x A$. Writing $z=x b_{0}-y$, we have $\bar{y} \bar{z} \neq 0$ (else $y=x \bar{y}$ ). By Lemma 1 applied to $A(\bar{y})$, there exists $b_{1} \in A(\bar{y})$ such that (i) $\mu(y a) \leqq \mu(x a) / 2$ whenever $a$ $\in A\left(b_{1}\right)$, (ii) $\mu(y a)>\mu\left(x a^{\prime}\right) / 2$ whenever $a^{\prime} \in A\left(y-b_{1}\right)$ and $a^{\prime} \neq o$. If $b_{1} \neq o$, (2) is satisfied if we take $y_{1}=y b_{1}$. Hence we may assume $b_{1}=o$, and therefore, on taking $a^{\prime}=\bar{y} \bar{z}$, we have $\mu\left(y a^{\prime}\right)>\mu\left(x a^{\prime}\right) / 2$. A similar argument using $z$ gives $\mu\left(z a^{\prime}\right)>\mu\left(x a^{\prime}\right) / 2$; and, adding, we obtain a contradiction.

By iteration, the factor $1 / 2$ in (2) can be replaced by $(1 / 2)^{n}$, and from (1) the lemma is established in the case $\mu(x)<\infty$.

Now suppose $\mu(x)=\infty$. It is easy to see that there will be a maximal element $b_{\infty} \in A$ such that $f(a)=\infty$ whenever $a \in A\left(b_{\infty}\right)$ and $a \neq 0$. If $b_{\infty} \neq 0$, we have only to take $y=x b_{\infty}$. Hence we can assume $b_{\infty}=o$, and consequently can write $e=\mathrm{V} b_{n}, n=1,2, \cdots$, where the elements $b_{n}$ are disjoint, belong to $A$, and satisfy $f\left(b_{n}\right)<\infty$. Define $f_{n}$ by: $f_{n}(a)=f\left(a b_{n}\right), a \in A$; then $f=\sum f_{n}$, so that for at least one $n$ the function $f_{n}$ is not identically zero, and it will suffice to prove the lemma for $f_{n}$ instead of $f$. That is, we may assume that $f$ is finite.

From Lemma 1 there is a maximal element $b_{0} \in A$ for which $f\left(b_{0}\right)=0$. If $\mu\left(x-b_{0}\right)<\infty$, the lemma again follows, since we can replace $x$ by $x-b_{0}$. Hence we may assume $\mu\left(x-b_{0}\right)=\infty$. Since $\mu$ is $\sigma$-finite, we can write $x-b_{0}$ $=\bigvee x_{n}$, where $x_{1} \leqq x_{2} \leqq \cdots$ and $\mu\left(x_{n}\right)<\infty$. Since $f$ is finite, there must exist $n$ and a nonzero $b^{\prime} \in A\left(-b_{0}\right)$ such that $\mu\left(x_{n} a\right) \geqq f(a)$ whenever $a \in A\left(b^{\prime}\right)$. The lemma now follows from the finite-measure case, since we can replace $x$ by $x_{n}$ and $f$ by the function $f_{0}$ defined by: $f_{0}(a)=f\left(a b^{\prime}\right)$.

LEMma 3. Under the hypotheses of Lemma 2, there exists $y \leqq x$ such that $\mu(y a)=f(a)$ for all $a \in A$. 
This follows from Lemma 2 by an "exhaustion" argument.

3.5. From Lemma 3 it follows at once that $\sim$ satisfies postulate II of $[8$, p. 281]; and postulates 0 and I are here trivial. Postulate III also follows readily, in view of the facts that (i) elements of finite $\mu$ are bounded( $\left.{ }^{9}\right)$, (ii) equivalence is here subtractive (cf. $[8,6.1]$ ). Lemma 3 also shows that there are no nonzero indecomposable elements [8, p. 295]. To complete the proof of Theorem 1 we have only to show that the invariant algebra of $(E, \sim)$ is $A$.

Each $a \in A$ is invariant; for otherwise $[8,5.3]$ there would exist elements $x \leqq a, y \leqq-a$, such that $y \sim x \neq o$, whence $\mu(x)=\mu(x a)=\mu(y a)=0$, a contradiction.

Conversely, suppose $x$ is invariant but does not belong to $A$. Then the same is true of the elements $y=\bar{x}-x$ and $z=x-\mathrm{Cl}(-x)$. By Lemma 1 (3.4) there exists $b_{0} \in A$ such that $\mu(a y) \leqq \mu(a z)$ if $a \in A\left(b_{0}\right)$, and $\mu(a y)>\mu(a z)$ if $a \in A\left(-b_{0}\right)$ and $a \neq o$. From Lemma 3 (3.4) there is a sub-element of $z$ which is equivalent to $b_{0} y$, and since $y, z$ are disjoint and invariant it follows that $b_{0} y=o$. Similarly $z-b_{0}=o$. Thus $z \leqq b_{0}$, whence $x \leqq(-\mathrm{Cl}(-x)) \vee b_{0}$, so that $y \leqq \bar{x} \leqq(-\mathrm{Cl}(-x)) \bigvee b_{0}$; but $y$ is disjoint from $x$ and therefore from $-(\mathrm{Cl}(-x))$, so that $y=y b_{0}=o$, a contradiction.

3.6. The theorem of $[8,19.4]$ now gives (since there are here no indecomposable elements) an isometry $\phi$ between $(E, \sim)$ and the principal ideal of a certain element, $K$ say, in $(J, m) \times A$, where $(J, m)$ is a certain $(\sigma$-finite) numerical measure algebra. In order to derive an isometry of $(E, \mu)$ from this, we recall (cf. $[8, \S 10]$ ) that we have $e=e^{\prime} \bigvee e^{\prime \prime}$ where $e^{\prime}$ is the greatest bounded invariant element and $e^{\prime \prime}=\mathrm{V} f^{n}, n=1,2, \cdots$, to $\infty$, where the elements $f^{n}$ are disjoint, bounded and equivalent. Define a measure $\nu$ on $A$ by

$$
\nu(a)=\mu\left(a e^{\prime}\right)+\mu\left(a f^{1}\right),
$$

$a \in A$,

(note that $\mu\left(a f^{1}\right)=\mu\left(a f^{n}\right)$ because $\left.f^{1} \sim f^{n}\right)$. Obviously $\nu$ is positive and $\sigma$-additive, and we shall show below that $\nu$ is $\sigma$-finite on $A$. Taking this for granted, we assert that $\phi$ is an isometry of $(E, \mu)$ onto the ideal of $K$ in $(J, m) \times(A, \nu)$ that is, from 2.4 , that for the relevant sets $H$ in a realization of this product we have $\int M\{H\} d \nu=\mu\left(\phi^{-1}(H)\right)$. By the usual approximation argument, it will suffice to prove this for "rectangular" sets $\left({ }^{10}\right)$; thus we have only to show that, for $x \in J$ and $a \in E$, and either $a \leqq e^{\prime}$ or $a \leqq e^{\prime \prime}$, we have $\mu(x a)$ $=m(x) \nu(a)\left({ }^{11}\right)$. Suppose first $a \leqq e^{\prime}$. From the construction of $J$ (see $[8,19.4$ and 18.1]), we may further assume $x \leqq j_{n}=e_{n}$, and $a \leqq \mathrm{Cl}\left(e_{n}\right)$. Now, from the construction of $m$ (see $\left[8,17.1\right.$ and 8.1]) we have $[x a]=\left(m(x) / \rho_{n}\right)\left[a e_{n}\right]$ and $\left[a e_{n}\right]=\rho_{n}[a]$; thus $[x a]=m(x)[a]$, and from the definition of class-

$\left(^{9}\right)$ For the definition of "bounded," see $[8$, p. 281]. Note that bounded elements need not have finite $\mu$-measure; see 3.7 below.

(10) Since the element $K$ corresponds to a countable union of such rectangles, from the construction in $[8, \S \S 18,19]$.

(11) To simplify the notation, we have identified $J$ with the corresponding sub-algebra of $E$. 
multiplication $[8, \S 12]$ it readily follows that $\mu(x a)=m(x) \mu(a)=m(x) \nu(a)$, as desired. If $a \leqq e^{\prime \prime}$, we can assume $[8,19.1]$ that $x \leqq j^{n}$. Since the construction relative to $f^{n}$ was the same as for $E\left(e^{\prime}\right)$, we have as before $\mu(x a)$ $=m(x) \mu\left(a f^{n}\right)=m(x) \nu(a)$, completing the proof.

3.7. The $\sigma$-finiteness of $\nu$ on $A$ is an immediate consequence of the boundedness of $e^{\prime}$ and $f^{n}$, in view of the following lemma:

LEMMA 4. A necessary and sufficient condition that $x \in E$ be bounded in $(E, \sim)$ is that $x \leqq \mathrm{~V} b_{n}$, where $b_{n} \in A$ and $\mu\left(b_{n} x\right)<\infty$.

If the condition is satisfied, there is no loss in supposing the elements $b_{n}$ to be disjoint; each of the elements $b_{n} x$ is bounded, having finite $\mu$, and their invariant closures are disjoint, so that $[8,6.5] \mathrm{V} x b_{n}=x$ is bounded.

Conversely, suppose that $x$ does not satisfy the condition. Then there exists $y \leqq x$ such that $y \in x A$ and, for each nonzero $a \in A(\bar{y}), \mu(a y)=\infty$. Since $\mu$ is $\sigma$-finite on $E$, there exists $y_{1} \leqq y$ such that $0<\mu\left(y_{1}\right)<\infty$, and therefore, if $a \in A$ and $a y \neq 0, \mu\left(a\left(y-y_{1}\right)\right)=\infty=\mu(a y)$. Thus $y-y_{1} \sim y$. From postulate II of [8], there exists $y_{2} \leqq y-y_{1}$ such that $y_{2} \sim y_{1}$, and consequently $\mu\left(a\left(y-\left(y_{1} \bigvee y_{2}\right)\right)\right)=\infty=\mu(a y)$ if $a \in A$ and $a y \neq 0$, so that $y-\left(y_{1} \bigvee y_{2}\right) \sim y$. Iteration of this argument produces an infinite sequence of disjoint equivalent nonzero elements $y_{n} \leqq y$. Hence (from $[8,6.3]$ ) $y$, and so, a fortiori, $x$, is unbounded.

3.8. In 3.3-3.7, all considerations were relative to $e_{*}$. If we note that the relative algebra $A e_{*}$ is isomorphic to the ideal $A\left(\bar{e}_{*}\right)$ (cf. $[8,8.3]$ ), we see that the results of 3.6 and $3.2(7)$ can be combined into the following theorem.

THEOREM 2. Let $(E, \mu)$ be a ( $\sigma$-finite) measure algebra, with a given subalgebra $A$ (on which $\mu$ need not be $\sigma$-finite). Then there exist: a $\sigma$-finite measure algebra $(J, m)$, elements $b_{*}, b_{1}, b_{2}, \cdots$ of $A$, and $\sigma$-finite positive measures 0 , $\mu_{1}, \mu_{2}, \cdots$ on the respective ideals $A\left(b_{*}\right), A\left(b_{1}\right), A\left(b_{2}\right), \cdots$ such that:

(i) $b_{*} \bigvee b_{1}=e$, and $b_{1} \geqq b_{2} \geqq \cdots$.

(ii) $(E, \mu)$ is isometric, under an isometry $\psi$, to the direct sum of the measure algebras $\left(A\left(b_{n}\right), \mu_{n}\right)$ and of the principal ideal of an element $K$ in the direct product $(J, m) \times\left(A\left(b_{*}\right), \nu\right)$.

(iii) To each $a \in A, \psi$ assigns the element $a b_{n}$ of $\left(A\left(b_{n}\right), \mu_{n}\right)$ and the element $K \wedge(J \times a)$ of $(J, m) \times\left(A\left(b_{*}\right), \nu\right)$.

(iv) If $a \in A$ is expressible as $\mathrm{V} a_{n}$, where $a_{n} \in A$ and $\mu\left(a_{n}\right)<\infty$, then $\mu(a)$ $=\sum \mu_{n}\left(a b_{n}\right)+\nu\left(a b_{*}\right)$.

In fact, the elements $b_{n}, b_{*}$, will be the closures of $d_{n}(3.2(5))$ and $e_{*}$ respectively; the construction of $\psi$ is clear. The only statement requiring proof is the last, which follows from the definitions of $\mu_{n}$ and $\nu$ and the observation that $a$ must be bounded (Lemma 4, 3.7) and so disjoint from $e^{\prime \prime}$.

4. Algebraic decomposition theorems.

4.1. Two other ways of stating Theorem 2 deserve attention. In the first 
place, we may decompose $K$ into its constituent "rectangles" (following the construction in [8]), obtaining the following extension of a theorem of Nikodým [9].

Theorem 2a. Given a sub-algebra $A$ of a measure algebra $(E, \mu)$, there exist: elements $b^{1}, b^{2}, \cdots$ of $A, \sigma$-finite positive measures $\mu^{n}$ on the respective ideals $A\left(b^{n}\right)$, and measure algebras $\left(J_{n}, m_{n}\right)$, such that $E$ is isometric to the direct sum of the direct products $\left(J_{n}, m_{n}\right) \times\left(A\left(b^{n}\right), \mu^{n}\right)$. This isometry can be chosen so that it maps each $a \in A$ on the element $J_{n} \times a b^{n}$ of the nth direct product. Each $\left(J_{n}, m_{n}\right)$ is isometric to one of the following types:

(i) $I^{\mathfrak{m}}$ for some cardinal $\mathfrak{m}$ (depending on $\left.n\right)\left({ }^{12}\right)$,

(ii) the direct sum of $\boldsymbol{\aleph}_{0}$ copies of $I^{\mathrm{m}}$, or

(iii) the algebra generated by a single atom.

(The last type arises, of course, from the algebras $\left(A\left(b_{n}\right), \mu_{n}\right)$ in Theorem 2. Note that, while $\mathrm{V} b^{n}=e$, the elements $b^{n}$ will not in general be disjoint.)

REMARK. In particular cases, this somewhat cumbrous description may reduce considerably; for example, if $\mu(e)=1$ and $E$ is separable and has no elements of order 0 over $A$, it is clear from the construction in $\$ 3$ and [8] that only one summand survives, and we obtain that $(E, \mu)$ is isometric to $I^{1} \times(A, \mu)$, under an isometry which maps each $a \in A$ on itself.

4.2. Secondly, we may combine all the direct summands together. The result is:

Theorem 2b. Given a sub-algebra $A$ of a measure algebra $(E, \mu)$, there exist $a \sigma$-finite positive measure $\nu$ on $A$ and a measure algebra $\left(J^{0}, m^{0}\right)$, such that there is an isometry of $(E, \mu)$ onto a sub-algebra of a principal ideal, say of $K^{0}$, of the direct product $\left(J^{0}, m^{0}\right) \times(A, \nu)$, which maps each $a \in A$ on the element $K^{0} \wedge\left(J^{0} \times a\right)\left({ }^{13}\right)$.

REMARK. If $E$ contains no elements of order 0 over $A$, Theorem 2 shows that the phrase "sub-algebra of a principal ideal" can be replaced by "principal ideal" here; but it is easy to show by counterexamples that this replacement is not possible in general.

To prove Theorem $2 \mathrm{~b}$, it will be enough to imbed $\left(E\left(e_{0}\right), \mu\right)$ in this way; for in 3.6 we obtained an isometry of $\left(E\left(e_{*}\right), \mu\right)$ on a principal ideal in a direct product $(J, m) \times\left(A\left(\mathrm{Cl}\left(e_{*}\right)\right), \nu\right)$, and the two isometries can be combined in a routine way. We extend the definition of $\nu$ (from $A\left(\bar{e}_{*}\right)$ to all of $A$ by setting, for each $a \in A$,

$$
\nu(a)=\nu\left(a \bar{e}_{*}\right)+\mu\left(a-\bar{e}_{*}\right)
$$

(12) $I^{\mathfrak{m}}$ denotes the direct product of $\mathfrak{m}$ copies of the measure algebra $I^{1}$ formed by the measurable sets modulo null sets of the unit interval.

(13) This is essentially a result announced by A. M. Gleason, Bull. Amer. Math. Soc. vol. 55 (1949) p. 283; but see the remark following. 
Since $e-\bar{e}_{*} \leqq d_{1}(3.2(5))$, an element of order $0,3.2(3)$ shows that $\nu$ is $\sigma$-finite on $A$; and clearly $\nu$ is a positive measure on $A$. For each $n$, the additive function $\mu\left(a d_{n}\right)$ on $A$ has a Radon-Nikodým representation, $\mu\left(a d_{n}\right)=\int f_{n} d \nu$, the integral being taken over the open-closed subset $a^{*}$ (corresponding to $a \in A$ ) of the representation space $R$ of $A$. Here the real-valued function $f_{n}$ on $R$ can be taken to be continuous (cf., for example, $[8,4.2(7)]$ ); and, by an easy "exhaustion" argument, it follows that there exist positive constants $k_{n i}$ and elements $b_{n i} \in A$ such that, except for a set of first category in $R$, $f_{n}=\sum_{i} k_{n i} \chi\left(b_{n i}^{*}\right), \chi$ denoting the characteristic function. Integration gives

$$
\mu\left(a d_{n}\right) \equiv \mu_{n}(a)=\sum_{i} k_{n i} \nu\left(a b_{n i}\right), \quad \text { for all } a \in A .
$$

Let $\left(J_{0}, m_{0}\right)$ be the measure algebra generated by a sequence of atoms $\alpha_{n i}$, with $m_{0}\left(\alpha_{n i}\right)=k_{n i}$. Define a mapping $\phi$ of $E\left(e_{0}\right)$ in the product $\left(J_{0}, m_{0}\right)$ $\times(A, \nu)$ (realized by the product of the representation spaces of $J_{0}$ and $A$ ) by:

$$
\phi(x)=\bigcup_{n, i}\left[\alpha_{n i} \times\left\{\mathrm{Cl}\left(x d_{n}\right)^{*} \cap b_{n i}^{*}\right\}\right] \quad\left(x \leqq \mathrm{~V} d_{n}=e_{0}\right)
$$

It is easy to see that $\phi$ is an algebraic isomorphism of $E\left(e_{0}\right)$ onto a sub-algebra of a principal ideal in this product. Further, the measure of $\phi(x)$ is

$$
\sum_{n, i} k_{n i} \nu\left\{b_{n i} \mathrm{Cl}\left(x d_{n}\right)\right\}=\sum_{n} \mu\left\{d_{n} \mathrm{Cl}\left(x d_{n}\right)\right\}=\sum \mu\left(x d_{n}\right)
$$

(from $3.2(3))=\mu(x)$, establishing that $\phi$ is an isometry.

4.3. Finally, we remark that Theorem 2 includes a theorem of Dieudonné [3], as formulated by Halmos [5], without the restriction that the measure be finite, and gives moreover an analysis of the structure of the measures $\mu_{z}$ on the "fibres" $\pi^{-1}(z)\left({ }^{14}\right)$. Roughly speaking, each $\pi^{-1}(z)$ can be split into two parts (possibly empty), on one of which the measure $\mu_{z}$ is purely atomic, while the other parts can be derived from a direct product fibring. A brief indication of the argument is as follows. Let $Y$ be the representation space of $(E, \mu)$, and $Z$ that of $(A, \nu)$, where $A$ is a given sub-algebra of $E$ and $\nu$ is the $\sigma$-finite measure constructed in 4.2. From Theorem 2 (3.8), the openclosed subset $e_{*}^{*}$ of $Y$ may be regarded as an open-closed subset of the representation space $X$ of $J \times A$. On $e_{*}^{*}$ the natural mapping $\pi$ of $Y$ on $Z$ can be factored into (i) a natural mapping $\pi^{\prime}$ from $X$ to the product $R \times Z$ of the representation spaces of $J$ and $A$, followed by (ii) the projection $\pi^{\prime \prime}$ from $R \times Z$ on $Z$. A measure $\mu_{z}^{\prime}$ is defined on $e_{*}^{*} \cap \pi^{-1}(z)$ by taking $\mu_{z}^{\prime}\left\{\pi^{\prime-1}\left(j^{*} \times z\right)\right\}=m(j)$, $j \in J$. Again, $Y-e_{*}^{*}=\bigcup d_{n}^{*} \cup N$, where $N$ is nowhere dense; from 3.1(3) it follows that $\pi^{-1}(z)$ meets $d_{n}^{*}$ in at most one point, say $p_{n}(z)$. A measure $\mu_{z}^{0}$ is defined on $\left(Y-e_{*}^{*}\right) \cap \pi^{-1}(z)$ by giving the points $p_{n}(z)$ appropriate weights; and finally $\pi^{-1}(z)$ is made into a measure space, with measure $\mu_{z}$, by taking

(14) For the notations in this paragraph, see [5]. 
the direct sum of $\left(e_{*}^{*} \cap \pi^{-1}(z), \mu_{z}^{\prime}\right)$ and $\left(\pi^{-1}(z)-e_{*}^{*}, \mu_{z}^{0}\right)$. It is easy to verify that, for each $x \in E$, corresponding to $x^{*} \subset Y$, we have $\mu(x)=\int \mu_{z}\left(x^{*} \cap \pi^{-1}(z)\right) d \nu$, as required.

\section{Galois-sub-algebras.}

5.1. The principal application of the preceding algebraic decomposition theory will be to the case in which the given sub-algebra $A$ of $E$ consists of all elements of $E$ left invariant by some group of (measure-preserving) automorphisms of $(E, \mu)$. It is thus of some interest to characterize those subalgebras $A$ which are "Galois" - that is, which can arise in this way. The problem can be reformulated as follows. Given a measure algebra $(E, \mu)$, and a sub-algebra $A$ of $E$, let $\Phi$ be the group of all automorphisms $\phi$ of $(E, \mu)\left({ }^{15}\right)$ such that $\phi(a)=a$ for each $a \in A$, and let $A^{\prime}$ be the set of all elements $x \in E$ such that $\phi(x)=x$ for each $\phi \in \Phi$. Clearly $A^{\prime}$ is a sub-algebra of $E$, $A^{\prime} \supset A$, and $A$ is Galois if and only if $A^{\prime}=A$.

5.2. Theorem 3. Necessary and sufficient conditions that $A$ be Galois are: (i) for each infinite cardinal $\mathrm{m}, A$ contains the greatest element of $E$ which is homogeneous of order $\mathfrak{m}$ over $A[8, \S 11]$, and (ii) whenever $c, c^{\prime}$ are of order 0 over $A$, and $\bar{c}=\bar{c}^{\prime}(3.2)$, then $\mu(c)=\mu\left(c^{\prime}\right)$.

Necessity. Condition (i) is obviously necessary. To prove (ii) necessary, consider $\left(E, \sim_{\Phi}\right)[8,20.4]$; its invariant elements form precisely $A^{\prime}=A$, so that $\bar{x}(x \in E)$ has the same meaning here as in [8]. There exist $[8,7.5]$ elements $d \leqq c, d^{\prime} \leqq c^{\prime}$, such that $d \sim_{\Phi} d^{\prime}$ and $\mathrm{Cl}(c-d) \mathrm{Cl}\left(c^{\prime}-d^{\prime}\right)=o$. We have therefore $\mathrm{Cl}(c-d)=\bar{c} \mathrm{Cl}(c-d)=\mathrm{Cl}\left(c^{\prime}\right) \mathrm{Cl}(c-d)=\mathrm{Cl}\left(d^{\prime}\right) \mathrm{Cl}(c-d)=\bar{d} \mathrm{Cl}(c-d)$ $=o$, from 3.2(3); hence $d=c$, and similarly $d^{\prime}=c^{\prime}$. Thus $c \sim_{\Phi} c^{\prime}$, and a fortiori $\mu(c)=\mu\left(c^{\prime}\right)\left({ }^{16}\right)$.

Sufficiency. Given $x \in A^{\prime}$, and that (i) and (ii) hold, we shall prove $x=\bar{x}$ (and so $x \in A$ ). From (i), it will suffice to prove this assuming either (1) $x \leqq e_{0}$ (see $\S 3$ ), or (2) $x \leqq a_{\mathfrak{m}}$, where $a_{\mathfrak{m}}$ is the greatest element homogeneous of infinite order $\mathfrak{m}$ over $A$ (so that $a_{\mathfrak{m}} \in A$ ).

(1) Suppose $x \leqq e_{0}=\bigvee d_{n}$, but $x \neq \bar{x}$. Write $x_{n}=x d_{n}$; then $\bar{x}=\bigvee \bar{x}_{n}$, and so, for some $n,(\bar{x}-x) \bar{x}_{n} \neq o$. It follows that, for some $k$, we have $(\bar{x}-x) \bar{x}_{n} d_{k}=t$ say $\neq o$. Since $\bar{t} x_{n} \leqq d_{n}, \bar{t} x_{n}$ is of order 0 , and so (3.2(3)) $E\left(\bar{t}_{n}\right)$ is (algebraically) isomorphic to $A(\bar{t})$, under the correspondence $s \rightarrow \bar{s}\left(s \leqq \bar{t} x_{n}\right)$. Similarly, $t$ is of order 0 and $A(\bar{t})$ is isomorphic to $E(t)$ under the correspondence $a \rightarrow t a$ $(a \in A(\bar{t}))$. Define $\psi(s)=t \bar{s}$; thus $\psi$ is an isomorphism of $E\left(\bar{t} x_{n}\right)$ onto $E(t)$. Further, if $s \leqq \bar{t} x_{n}$, (ii) shows that $\mu(s)=\mu(t \bar{s})$, so that $\psi$ is measure-preserving. Now $t$ and $\bar{t} x_{n}$ are disjoint; hence the mapping defined by

$$
\phi(y)=\left\{y-\left(\bar{t} x_{n}\right)-t\right\} \vee \psi\left(y \bar{t} x_{n}\right) \vee \psi^{-1}(y t), \quad y \in E,
$$

is a mapping in the group $\Phi$ such that $\phi(x) \neq x$, which contradicts $x \in A^{\prime}$.

(15) It is, of course, understood that each $\phi \in \Phi$ is measure-preserving.

(16) It follows further that $a c \sim a c^{\prime}$, and so $\mu(a c)=\mu\left(a c^{\prime}\right)$, for each $a \in A$. 
(2) Suppose $x \leqq a_{\mathfrak{m}}, x \in A^{\prime}$, but $x \neq \bar{x}$. It will evidently suffice to consider the ideals $E\left(a_{\mathfrak{m}}\right), A\left(a_{\mathfrak{m}}\right)$, only; thus we may here suppose that $a_{\mathfrak{m}}=e$. We apply the argument of 3.3-3.7, defining $\sim$ as in 3.3(1), and have $e=e^{\prime} \vee e^{\prime \prime}$ where these are disjoint elements of $A$ (e' being the greatest bounded element of $A)$. It will suffice to assume $x \leqq e^{\prime}$ or $x \leqq e^{\prime \prime}$, and to consider the ideals $E\left(e^{\prime}\right), E\left(e^{\prime \prime}\right)$, separately. Because $e^{\prime}$ and $e^{\prime \prime}$ are now homogeneous, the construction in $[8, \S \S 18,19]$ reduces, and we have $\left(E\left(e^{\prime}\right), \sim\right)$ isomorphic to the abstract product $(J, m) \times A\left(e^{\prime}\right)$, where $(J, m)$ is isometric to $I^{\mathfrak{m}}$. A similar statement applies to $\left(E\left(e^{\prime \prime}\right), \sim\right)$, except that $(J, m)$ is here isometric to the direct sum of $\boldsymbol{\aleph}_{0}$ copies of $I^{\mathfrak{m}}$. The proof in $\$ 3$ shows that $\left(E\left(e^{\prime}\right), \mu\right)$ is isometric to $(J, m) \times\left(A\left(e^{\prime}\right), \nu\right)$, with a similar statement for $e^{\prime \prime}$. Thus there is no loss of generality in taking $(E, \mu)$ to be simply $(J, m) \times(A, \nu)$.

Since $x \neq \bar{x}$, there exist $[8,5.3]$ nonzero elements $y \leqq e-x, z \leqq x$, such that $y \sim z$; and we can further suppose $\mu(y)(=\mu(z))<\infty$. Consider the measurefunctions $M\{y\}, M\{z\}$, in the abstract product $(J, m) \times A$; these are equal, finite, continuous non-negative functions on the representation space $R$ of $A$, and are not identically 0 . Hence there exists a nonzero $a_{0} \in A$ such that, for each $p \in a_{0}^{*}, M\{y\}(p)=M\{z\}(p)>\epsilon>0$. We can approximate to $y$ and $z$ by countable "rectangle-sums" $r=\bigvee r_{i} a_{i}, s=\bigvee s_{i} a_{i}\left(r_{i}, s_{i} \in J, a_{i} \in A, \bigvee a_{i}=e\right.$, $a_{i} a_{j}=0$ if $\left.i \neq j, i, j=1,2, \cdots\right)$, such that $M\left\{y+{ }_{2} r\right\}<\epsilon / 6$ and $M\left\{z+{ }_{2} s\right\}$ $<\epsilon / 6$ for all $p \in R$ (cf. $[8,4.6]$ ). For some $i$, we have $a_{i} a_{0} \neq o$. Choose $b \in A$ so that $o<b \leqq a_{i} a_{0}$ and $\nu(b)<\infty$; thus, for each $p \in b^{*}$ we have $M\left\{y+{ }_{2} r_{i} b\right\}$ $<\epsilon / 6$ and $M\left\{z+{ }_{2} s_{i} b\right\}<\epsilon / 6$. Since $M\{y\}=M\{z\}$, it follows that $\mid m\left(r_{i}\right)$ $-m\left(s_{i}\right) \mid<\epsilon / 3$; hence, on replacing $r_{i}$ or $s_{i}$ by a smaller element of $J$, we obtain elements $h, k \in J$ such that $m(h)=m(k)<\infty$, and (from 2.4) $\mu(h b-y b$ ) $<\epsilon \nu(b) / 2$ and $\mu(z b-k b)<\epsilon \nu(b) / 2$.

From the structure of $J$, there exists a (measure-preserving) automorphism of $(J, m)$ which interchanges $h$ and $k$; and this is readily extended to give an automorphism $\phi$ of $(J, m) \times(A, \nu)=(E, \mu)$, such that $\phi(a)=a$ whenever $a \in A, \phi(h)=k$, and $\phi(k)=h$. Thus $\phi(h b)=k b$ and $\phi(k b)=h b$. Write $t=z k b \phi(y h b)$; since $t \leqq z \leqq x$ and $\phi^{-1}(t) \leqq y \leqq e-x$, the assumption $x \in A^{\prime}$ gives $t=0$. Thus $z k b \leqq k b-\phi(y h b)=\phi(h b-y)$, so $\mu(z k b) \leqq \mu(h b-y)$ $<\epsilon \nu(b) / 2$, and therefore $\mu(z b)<\epsilon \nu(b)$. But $\mu(z b) \geqq \epsilon \nu(b)$, from 2.4 and the choice of $a_{0}$; and this contradiction completes the proof of Theorem 3 .

5.3. On combining Theorem 3 with Theorem $2 \mathrm{a}(4.1)$, we see that for Galois sub-algebras $A$ the decomposition of $E$ over $A$ can be simplified. We do not state the resulting theorem, since it is precisely the theorem of $[8$, 20.4]. Further, if $A$ is the algebra of elements invariant under a group $\Phi$ of automorphisms of $(E, \mu)$, the equivalence relations $\sim(3.3(1))$ and $\sim_{\Phi}[8$, 20.4 ] coincide. This simplification is moreover characteristic of Galois subalgebras; in fact, if a decomposition of the type of $[8,20.4]$ exists (where $A$ is the direct sum of the relative algebras $B_{n}$, in the notation of [8]), it is easy to see that the conditions of Theorem 3 are satisfied, so that $A$ is Galois. 


\section{Point-set decompositions.}

6.1. We next consider some "point-set" consequences of the preceding algebraic decomposition theory. To derive the analogous results for measure spaces, we shall have to assume that they are separable and in fact normal $\left({ }^{17}\right)$. It is convenient, however, to extend the meaning of "normal" to allow the measure to become infinite (but $\sigma$-finite), and to allow atoms. Thus we define a measure space to be $\sigma$-normal if it is almost isometric (cf. 1.2) to the direct sum of (i) a measure space formed by a countable number of atomic points, of positive finite weights, and (ii) an interval, possibly infinite, on the real line.

Theorem 4 below will be concerned with a strictly separable $\left({ }^{18}\right)$ field $\mathcal{A}$ of measurable subsets of a $\sigma$-normal measure space $(\Omega, \mu)$. Before we state it, consider the following examples (which will be proved to be typical):

(1) $\Omega_{a}$ is the direct sum of a countable number of disjoint $\sigma$-normal measure spaces $\left(Y_{n}, \mu_{n}\right)$, and $\mathcal{A}_{a}$ is the field generated by the sets $Y_{n}$.

(2) $\Omega^{\beta}$, conveniently regarded as situated in the plane, with coordinates $(\xi, \eta)$, consists of (i) a rectangle $0<\xi<\alpha, 0<\eta<\beta$, with ordinary plane measure, together with (ii) a countable number of line segments $\eta=-n, 0<\xi<\alpha$, $n=1,2, \cdots$, each with an absolutely continuous measure $\mu_{n}$ (which may vanish for a non-null set of $\xi$ 's). Here $\alpha$ may be finite or infinite; in the applications, $\beta$ will be 0,1 , or $\infty$. The field $A^{\beta}$ consists of all sets of the form $\Omega^{\beta} \cap \mathrm{Cyl}(H)$, where $H$ is a Borel set of $\xi^{\prime}$ 's and Cyl $(H)$ denotes the set of all points $(\xi, \eta)$ with $\xi \in H\left({ }^{19}\right)$.

6.2. Theorem 4. Given a strictly separable field $\mathcal{A}$ of measurable sets in a $\sigma$-normal measure space $(\Omega, \mu)$, then, after discarding a null $\mathcal{A}$-set,$(\Omega, \mu)$ is isometric to a direct sum $\Omega_{a} \oplus \Omega^{0} \oplus \Omega^{1} \oplus \Omega^{\infty}$, under an isometry which maps $A$ isomorphically onto $A_{a} \oplus \mathcal{A}^{0} \oplus \mathcal{A}^{1} \oplus \mathcal{A}^{\infty}$.

Proof. Let $(E, \mu)$ be the measure algebra associated with $(\Omega, \mu)$, and let $A$ be the sub-algebra of $E$ which corresponds to $\mathcal{A}$. Let $a_{1}, a_{2}, \cdots$ be the atoms of $A$ (necessarily countable); from the existence of a countable basis in $\mathcal{A}$ it readily follows that there is a smallest $\mathcal{A}$-set, say $Y_{n}$, in the class of $a_{n}$, and we have only to take $\Omega_{a}=U\left(Y_{n}, \mu\right)$. In what follows we can therefore assume that $A$ is non-atomic.

We apply Theorem $2 \mathrm{a}(4.1)$, noting that $E$ is now separable; thus the decomposition reduces to

${ }^{\left({ }^{17}\right)}$ For the definition and properties of normal measure spaces, see [6]. Some hypothesis similar to normality is essential for the theorems which follow (cf. $[3$, p. 42]). As Dieudonné has shown [3, pp. 40,51], normality may be avoided by using representation space realizations (as in 4.3 above); the resulting theorems are then essentially measure-algebraic in character.

(18) A field $\mathcal{A}$ is strictly separable $[4$, p. 387] if it is generated by countably many sets of $A$. As pointed out in [4], this assumption on $A$ involves no essential loss of generality here.

(19) If $\beta=0$, the definition of $\Omega^{0}$ should be modified to permit the omission, for each $n$, of a Borel subset of the line $\eta=-n$ having zero $\mu_{n}$-measure. 


$$
e=\bigvee d_{n} \vee e^{\prime} \vee e^{\prime \prime},
$$

where these elements are disjoint, $d_{n}$ is of order 0 over $A,\left(E\left(d_{n}\right), \mu\right)$ is isometric to $\left(E\left(\mathrm{Cl}\left(d_{n}\right)\right), \mu_{n}\right),\left(E\left(e^{\prime}\right), \mu\right)$ is isometric to $(I, m) \times\left(A\left(\mathrm{Cl}\left(e^{\prime}\right)\right), \nu\right)$, and $\left(E\left(e^{\prime \prime}\right), \mu\right)$ is isometric to $(J, m) \times\left(A\left(\mathrm{Cl}\left(e^{\prime \prime}\right)\right), \nu\right)$, where $(I, m)$ and $(J, m)$ are respectively the measure algebras associated with the unit interval (that is, $\left.I^{1}\right)$ and with an infinite interval. Further, $e^{\prime}$ and $e^{\prime \prime}$ are invariant relative to $e-\mathrm{V} d_{n}$, from which it readily follows that $\mathrm{Cl}\left(e^{\prime}\right) \mathrm{Cl}\left(e^{\prime \prime}\right)=o$.

We can thus choose disjoint measurable sets $D_{n}, E^{\prime}, E^{\prime \prime}$, in the respective classes $d_{n}, e^{\prime}, e^{\prime \prime}$, and disjoint sets $F^{\prime} \in \mathcal{A}, F^{\prime \prime} \in \mathcal{A}$, corresponding to $\mathrm{Cl}\left(e^{\prime}\right)$ and $\mathrm{Cl}\left(e^{\prime \prime}\right)$, in such a way that $E^{\prime} \subset F^{\prime}$ and $E^{\prime \prime} \subset F^{\prime \prime}$.

There is a set-isometry $\phi$ between $\left(E\left(e^{\prime}\right), \mu\right)$ and the rectangle $0<\xi<\alpha$ $=\nu\left(\mathrm{Cl}\left(e^{\prime}\right)\right), 0<\eta<1$, which induces a set-isometry between $\left(A\left(\mathrm{Cl}\left(e^{\prime}\right)\right), \nu\right)$ and the "cylinder sets" of unit height based on measurable $\xi$-sets in $0<\xi<\alpha$. Thus $\phi$ induces a set-isometry $\theta$ between $\left(A\left(\mathrm{Cl}\left(e^{\prime}\right)\right), \nu\right)$ and the linear interval (possibly infinite) $0<\xi<\alpha$. Again, there is a set-isometry $\phi_{n}$ between $\left(E\left(d_{n} \bar{e}^{\prime}\right), \mu\right)$ and $\left(A\left(\bar{d}_{n} \bar{e}^{\prime}\right), \mu_{n}\right)$ (in fact, $\left.\phi_{n}(x)=\bar{x}\right)$; thus $\theta \phi_{n}$ gives a set-isometry between $\left(D_{n} \cap F^{\prime}, \mu\right)$ and the interval $0<\xi<\alpha, \eta=-n$, if we use the absolutely continuous measure $\mu_{n}$ on the latter. Together, $\phi$ and $\theta \phi_{n}(n=1,2, \cdots)$ constitute a set-isometry $\Phi$ between $\left(F^{\prime}, \mu\right)$ and the measure space $\Omega^{1}$ hereby constructed, which maps each $a \in A\left(\bar{e}^{\prime}\right)$ on $\Omega^{1} \cap$ Cyl $(\theta(a))$, modulo null sets.

Since $F^{\prime}$ and $\Omega^{1}$ are both $\sigma$-normal, $\Phi$ can be realized by a point-isometry $\Psi$ of $F^{\prime}-N^{\prime}$ on $\Omega^{1}-N^{1}$, where $N^{\prime}$ and $N^{1}$ are null [6, Theorem 3 ].

Now, since $A$ is strictly separable, there exist a countable collection of $A$-sets, say $S_{\rho}$ (where $\rho$ runs over all rational values with $0 \leqq \rho<\alpha$ ), and a null $\mathcal{A}$-set $T \subset F^{\prime}$, such that (i) $S_{\rho} \subset F^{\prime}-T$, (ii) $S_{0}=0$, and $S_{\rho} \subset S_{\sigma}$ whenever $\rho<\sigma$, (iii) $\nu\left(S_{\rho}\right)=\rho$, and (iv) the sets $S_{\rho}$ generate all the $\mathcal{A}$-sets in $F^{\prime}-T$. (Cf. $\left[10\right.$, p. 602] and $[8,14.2-14.4]$.) For each $p \in F^{\prime}-T$, define $\Xi(p)$ $=\inf \left\{\rho \mid S_{\rho} \ni p\right\}=\sup \left\{\rho \mid S_{\rho} \boxplus p\right\}$ It is easy to see that $\Xi$ and $\Xi^{-1}$ map $\mathcal{A}$-sets in $F^{\prime}-T$ onto Borel $\xi$-sets, and conversely. There is no loss in assuming that the set-mapping $\theta$ maps each $S_{\rho}$ onto the interval $(0, \rho)$; thus $\Psi\left(S_{\rho}\right)$ differs from the intersection of $\Omega^{1}$ with the cylinder on $(0, \rho)$ by a null set. Hence, after enlarging the null sets $N^{\prime}$ and $N^{1}$, we may suppose that $N^{\prime} \supset T$, that $N^{\prime} \supset \Omega^{1}-\Xi\left(F^{\prime}-T\right)$, and that the abscissa of $\Psi(p)$ is $\Xi(p)$ for all $p \in F^{\prime}-N^{\prime}$. Let $H_{1}$ be the set of $\xi$ 's for which the segment $\xi=$ constant, $\eta>0$, meets $N^{1}$ in a set of positive (linear) outer measure; thus $H_{1}$ is null, and so is contained in a null Borel $\xi$-set $H_{2}$. Define $\Omega^{\prime}=\Xi^{-1}\left(\Omega^{1}-\mathrm{Cyl}\left(H_{2}\right)\right)$; thus $\Omega^{\prime}$ is an $\mathcal{A}$-set, $\Omega^{\prime} \subset F^{\prime}$, and $\mu\left(F^{\prime}-\Omega^{\prime}\right)=0$. For each $p \in \Omega^{\prime}$, the set $\Xi^{-1}(\Xi(p))$ (the smallest $A$-set containing $p)$ is mapped by $\Psi$, except for its intersection with $N^{\prime}$, onto the set $\Omega^{\prime} \cap\{\xi=\Xi(p)\}$, except for its intersection with $N^{1}$; but the latter intersection is (linearly) null, and so $\Psi$ can be redefined (altering it on null sets only) so as to map $\Xi^{-1}(\Xi(p))$ onto $\Omega^{\prime} \cap\{\xi=\Xi(p)\}$. The abscissa of $\Psi(p)$ is now $\Xi(p)$, for all $p \in \Omega^{\prime}$; hence $\Psi$ is an isometry of $\Omega^{\prime}$ onto $\Omega^{1}-\operatorname{Cyl}\left(H_{2}\right)$ 
which maps the $A$-sets of $\Omega^{\prime}$ onto Borel cylinder sets in $\Omega^{1}-\mathrm{Cyl}\left(H_{2}\right)$, and conversely. Finally, the complement of $H_{2}$ in the interval $(0, \alpha)$ can be mapped isometrically on the whole interval $(0, \alpha)$ by a mapping which preserves Borel sets $\left({ }^{20}\right)$; and the desired mapping of $\Omega^{\prime}$ on $\Omega^{1}$ has been constructed.

The argument mapping $F^{\prime \prime}$, except for a null $A$-set, onto $\Omega^{\infty}$ is completely similar, since the set-mapping between $\left(E\left(e^{\prime \prime}\right), \mu\right)$ and the rectangle $0<\xi<\nu\left(\bar{e}^{\prime \prime}\right), \eta>0$, can be taken to map each $f^{n}$ (cf. 3.6) on the strip $n-1$ $<\eta \leqq n$. And in $\Omega-F^{\prime}-F^{\prime \prime}$ the construction is simpler, as we can start with an arbitrary set-mapping $\theta$ of $\left(A\left(e-\bar{e}^{\prime}-\bar{e}^{\prime \prime}\right), \nu\right)$ on a linear interval.

6.3. REMARKs. It follows that, under the hypotheses of Theorem 4, every null set which is a union (not necessarily countable) of $A$-sets is contained in a null $\mathcal{A}$-set. Further, if a measure space is formed by taking the irreducible ("atomic") $A$-sets as points, and taking the field of sets derived (in an obvious way) from the $\mathcal{A}$-sets to be measurable, with measure derived from $\nu$, then the completion of this measure space (with respect to null sets) is $\sigma$-normal.

6.4. From Theorem 4 it is easy to deduce a theorem of Halmos [ 4 and 1; cf. also 3 and 5], to the effect that, under the hypotheses of Theorem 4, $(\Omega, \mu)$ is (except for a null $\mathcal{A}$-set) a direct sum of measure spaces $\left(Y_{\xi}, \mu_{\xi}\right)$ over a complete measure space $(X, X, \nu)$ in such a way that $X$ coincides with the "completion" of $A$, in a natural sense (cf. 5.3). In fact, one has only to take the "fibres" $Y_{\xi}$ to be (i) the spaces $Y_{n}$ in $\Omega_{a}$ (cf. 4.1), (ii) the intersections, for almost all $\xi$ 's, of the lines $\xi=$ constant with the spaces $\Omega^{0}, \Omega^{1}, \Omega^{\infty}$; the basespace $X$ and the measures involved are obvious. However, Theorem 4 gives rather more than the existence of a direct sum decomposition, since it shows that if we disregard the atomic parts of the "fibres" $Y_{\xi}$, the fibring is that of two direct products (or a single direct product if $\mu$ is finite). (Cf. 4.3.) As we now show, a similar sharpening holds, under reasonable conditions, for a given direct sum decomposition.

First we define a "standard" direct sum, as follows. Let $\left(X_{s}, \nu\right)$, the basespace, be an arbitrary $\sigma$-normal measure space, and let $f$ be a non-negative numerical measurable function on $X_{s}$; the value $\infty$ for $f$ is not excluded. Let $\left\{g_{n}\right\}$ be a sequence of non-negative finite measurable functions on $X_{8}$. Let $Y_{\xi}$, for each $\xi \in X_{8}$, consist of the interval $0<\eta<f(\xi)$, with ordinary measure, together with atoms of weights $g_{n}(\xi)$ at the points $\eta=-n$ whenever $g_{n}(\xi)>0$. The "standard" direct sum $\Omega_{8}$ is then the subset of the product of $X_{s}$ with the line $-\infty<\eta<\infty$ consisting of (i) the ordinate set $\left\{\xi \in X_{s}, 0<\eta<f(\xi)\right\}$, with ordinary product measure, and (ii) the sets $\left\{\xi \in X_{s}, g_{n}(\xi)>0, \eta=-n\right\}$, each with measure $\mu_{n}=\int g_{n} d \nu$.

${ }^{(20)}$ For simplicity we can suppose $H_{2}$ to be a $G_{\delta}$ set. Let $H_{3}$ be an uncountable null $G_{\delta}$ $\xi$-set disjoint from $\mathrm{H}_{2}$, and map $\mathrm{H}_{3}$ onto $\mathrm{H}_{2} \cup \mathrm{H}_{3}$ homeomorphically (see for example C. Kuratowski, Topologie I, Warsaw, 1933, p. 225). 
6.5. TheOREM 5. If a $\sigma$-normal measure space $(\Omega, \mu)$ is a direct sum of measure spaces $\left(Y_{x}, \mu_{x}\right)$ over a complete $\left({ }^{21}\right)$ ( $\sigma$-finite) measure space $(X, X, \lambda)$, and if $X$ has a separating sequence ${ }^{22}$, then there exist null sets $N_{1} \subset X, N_{2} \subset \Omega$, such that (i) if $x \in N_{1}, Y_{x} \subset N_{2}$, and if $x \notin N_{1}, \mu_{x}\left(Y_{x} \cap N_{2}\right)=0$, (ii) there exist isometries $\phi$ of $\Omega-N_{2}$ onto a standard direct sum $\Omega_{s}$, and $\theta$ of $X-N_{1}$ onto the base-space $X_{s}$, such that, for each $x \in X-N_{1}, \phi$ is an isometry of $\left(Y_{x}-N_{2}, \mu_{x}\right)$ onto the "standard" fibre $Y_{\theta(x)}$.

Sketch of proof. For each $Z \in X$, write $A(Z)=U\left\{Y_{x} \mid x \in Z\right\}$. The sets $A(Z)$ form a field $A$ which corresponds to a sub-algebra $A$ of the algebra $(E, \mu)$ associated with $\Omega$. As in 6.2 we construct a set-isometry, say $\psi$, of $(\Omega, \mu)$ on a measure space of the type considered in Theorem 4 ; this space is essentially a "standard" direct sum in which the function $f$ takes the values $0,1, \infty$, only. Further, $\psi$ maps $A$-sets on "cylinder sets" (modulo null sets). From $\sigma$-normality, $\psi$ can be realized by a point-isometry $\phi$, if we discard suitable null sets. Using the countable separating system, and discarding further null sets, we arrange that each $Y_{x}$ is mapped on a single "vertical" segment. Thus $\phi$ induces a point-mapping $\theta$ of $X$ on $X_{s}$; in the first instance, $\theta$ is not measure-preserving, but we change the measure in $X_{s}$ to make $\theta$ an isometry, at the same time adjusting the "ordinate-function" $f$ (and the functions $\left.g_{n}\right)$ to keep $\phi$ measure-preserving. The relation $\mu(U)=\int \mu_{x}\left(Y_{x} \cap U\right) d \lambda$, applied to a suitably chosen sequence of sets, is then used to show that (after discarding more null sets) $\phi$ is also an isometry of each $Y_{x}$. (In carrying out the detailed proof, it is convenient to dispose of the atoms in $X$ and in the spaces $Y_{x}$ separately.)

COROLlaRY. Under the hypotheses of Theorem 5 , the measure spaces $(X, \lambda)$ and (for almost all $x)\left(Y_{x}, \mu_{x}\right)$ are $\sigma$-normal.

\section{Decompositions of measure-preserving transformations.}

7.1. Let $\Phi$ be a group of isometries of a measure space $(\Omega, \mu)$ onto itself. An important special case of the preceding theory arises if we take $\mathcal{A}$ to be the field of sets $U$ which are invariant under $\Phi$ (that is, which satisfy $\phi(U)=U$ for each $\phi \in \Phi)$. From the algebraic point of view, a structural analysis of the behavior of $\Omega$ (modulo null sets) under $\Phi$ was given in [8, p. 328] (cf. also 5.3 above); we shall here deduce the analogous "point" theorems for $\sigma$-normal measure spaces, dealing in detail with the case in which $\Phi$ is generated by a single isometry $T$, and then indicating briefly the results for a flow. In what follows, it is understood that all sets considered are measurable.

If $X, Y$ are subsets of a measure space $(\Omega, m)$, on which $T$ is a given

(21) It is no restriction on the generality of a direct sum to require that its base-space be complete.

(22) A separating sequence in $\mathcal{X}$ is a sequence of sets $S_{n} \in \mathcal{X}$ such that, for every $p, q \in X$ with $p \neq q$, there exists $S_{i}$ containing $p$ but not $q$. 
isometry of $\Omega$ onto itself, we write $X \sim_{T} Y$ to mean that there exists a sequence of disjoint sets $X_{n}$ such that $m\left(\mathrm{U} X_{n}+{ }_{2} X\right)=0, m\left(\mathrm{U} T^{n}\left(X_{n}\right)+{ }_{2} Y\right)=0$, and the sets $T^{n}\left(X_{n}\right)$ are disjoint $(-\infty<n<\infty)$. Let $(E, m)$ be the measure algebra associated with $\Omega ; T$ can of course be regarded as an automorphism of $(E, m)$, and $\sim_{T}$ can be regarded as defined on $E$. An element $u \in E$ will be "invariant" if $T(u)=u$; any corresponding set $U \subset \Omega$ will be called "almost invariant." It is easy to see that these definitions of $\sim_{T}$ and invariance on $E$ agree with those in $[8,20.4]$. Trivially, an invariant set is almost invariant; conversely, it is easy to see that

(1) if $U$ is almost invariant, there exist invariant sets $U_{0}, U_{1}$, such that $U_{0} \subset U \subset U_{1}$ and $m\left(U_{1}-U_{0}\right)=0$.

7.2. THEOREM $6\left({ }^{23}\right)$. Let $T$ be an isometry of a $\sigma$-normal measure space $(\Omega, m)$ onto itself. There exist disjoint invariant sets $\Omega_{n}(n=1,2, \cdots)$ such that $m\left(\Omega-\cup \Omega_{n}\right)=0$, and $\sigma$-normal measure spaces $\left(X_{n}, \nu_{n}\right),\left(Y_{n}, \mu_{n}\right)$, such that:

(i) $\left(\Omega_{n}, m\right)$ is (to within an isometry) the direct product $\left(X_{n}, \nu_{n}\right) \times\left(Y_{n}, \mu_{n}\right)$.

(ii) For each $x \in X_{n}$, the set $x \times Y_{n}$ is invariant under $T$, and $T$ is an ergodic measure-preserving transformation on $\left(x \times Y_{n}, \mu_{n}\right)$.

(iii) For any $Q_{1}, Q_{2} \subset \Omega$, we have $Q_{1} \sim_{T} Q_{2}$ if and only if, for every $n$ and for almost every $x \in X_{n}$, we have $\mu_{n}\left\{\left(x \times Y_{n}\right) \cap Q_{1}\right\}=\mu_{n}\left\{\left(x \times Y_{n}\right) \cap Q_{2}\right\}$.

(iv) No two of the spaces $\left(Y_{n}, \mu_{n}\right)$ are isometric, and each of them is isometric to one of the following:

(a) the unit interval (with Lebesgue measure),

(b) the real line (with Lebesgue measure),

(c) a space consisting of $k(=k(n))$ atomic points $p_{1}, p_{2}, \cdots, p_{k}$, each of measure 1 (where $1 \leqq k \leqq \aleph_{0}$ ).

(v) If $\left(Y_{n}, \mu_{n}\right)$ is atomic (of type (c)), then, for each $x \in X_{n}, T\left(x \times p_{i}\right)$ $=\left(x \times p_{i+1}\right)$; here, if $k$ is finite, the suffixes $i, i+1$, are to be taken modulo $k$. Hence, if $k<\infty, T$ is periodic, with period $k$, on the corresponding set $\Omega_{n}$.

Proof. We apply the theorem of $[8$, p. 328$]$ to $(E, m)$ and the transformation group generated by $T$, obtaining a sequence of disjoint invariant elements $c_{n}$ such that $V c_{n}=e$, and with the further properties specified in that theorem. From 7.1(1) we can find disjoint invariant subsets $C_{n}$ of $\Omega$ in the respective classes $c_{n}$, and have $m\left(\Omega-\cup C_{n}\right)=0$. Each ideal $\left(E\left(c_{n}\right), m\right)$ is isometric to a direct product $\left(A_{n}, \mu_{n}\right) \times\left(B_{n}, \nu_{n}\right)$, where $A_{n}, B_{n}$ are sub-algebras of $E\left(c_{n}\right), B_{n}$ consisting of the invariant elements, and $\left(A_{n}, \mu_{n}\right)$ corresponding to one of the types listed in (iv) above. (It is convenient to take the factors in this product in the opposite order to that in the statement of Theorem 6.) As separable measure algebras, $\left(A_{n}, \mu_{n}\right)$ and $\left(B_{n}, \nu_{n}\right)$ can be realized by $\sigma$ normal measure spaces $\left(Y_{n}, \mu_{n}\right)$ and $\left(Z_{n}, \nu_{n}\right)$ respectively; and we thus have a set-isometry of $\left(C_{n}, m\right)$ onto $\left(Y_{n}, \mu_{n}\right) \times\left(Z_{n}, \nu_{n}\right)$, which can be realized by a

(23) Extending [4, Theorem 2] and [1, Theorem 2]. 
point-isometry $\phi_{n}$. In the first instance, $\phi_{n}$ is defined only on $C_{n}-0_{n}$, where $m\left(0_{n}\right)=0$, and $\phi_{n}\left(C_{n}-0_{n}\right)$ is only almost all of the product space; however, by altering $\phi_{n}$ on null sets and discarding a null invariant subset of $C_{n}$, we easily arrange that $\phi_{n}$ is an isometry of $C_{n}$ onto $Y_{n} \times Z_{n}$. In what follows, $\Omega_{n}$ will be derived from $C_{n}$, and $X_{n}$ from $Z_{n}$, by omitting suitable null sets; to simplify the notation we assume $n$ fixed and discard the suffix $n$, and take $\phi_{n}$ to be the identity mapping.

Let $\left\{S_{r}\right\}$ be a separating sequence in $Z$; the set $Y \times S_{r}$ corresponds to an element of the algebra $B$, and so is almost invariant. From 7.1(1), there exist invariant sets $U_{r}, V_{r}$, with $U_{r} \subset\left(Y \times S_{r}\right) \subset V_{r}$ and $m\left(U_{r}-V_{r}\right)=0$. Write $N_{1}=\mathrm{U}\left(V_{r}-U_{r}\right)$; thus $N_{1}$ is null and invariant, and it readily follows that, for each $x \in Z$, the set $(Y \times x)-N_{1}$ is invariant. Since (iv) of the present theorem is fulfilled, we may suppose first that $(Y, \mu)$ is an interval, finite or infinite; the atomic case will be considered later. Let $\left\{W_{r}\right\}$ be an enumeration of the rational intervals of $Y$ of finite lengths. Then, for every measurable set $S \subset Z$ with $\nu(S)<\infty$, we have $m\left[T\left\{\left(W_{r} \times S\right)-N_{1}\right\}\right]=\mu\left(W_{r}\right) \nu(S)$ $=m\left[T^{-1}\left\{\left(W_{r} \times S\right)-N_{1}\right\}\right]$; and, on applying Fubini's theorem, we readily obtain a null invariant set $N_{2} \supset N_{1}$ such that $T$ is an isometry on each $\left((Y \times x)-N_{2}, \mu\right)$. Further, by discarding a null set from $Z$ and an invariant null set from $C$, we may suppose that $\mu\left(N_{2} \cap(Y \times x)\right)=0$ for each $x \in Z$.

To obtain the ergodicity of $T$ on the sets $Y \times x$, we prove a superficially stronger (but equivalent) result: that, after discarding a suitable (fixed) null set of $x$ 's, we have, for every $Q_{1}, Q_{2} \subset Y$,

(1) $\mu\left(Q_{1}\right)=\mu\left(Q_{2}\right)$ if and only if $\left(Q_{1} \times x\right)-N_{2} \sim_{T, x}\left(Q_{2} \times x\right)-N_{2}$ where $\sim_{T, x}$ is defined as in 7.1 except that the measure space here considered is $((Y \times x)$ $\left.-N_{2}, \mu\right)$.

Let $W_{r}, W_{s}$ be any two rational intervals in $Y$ with $\mu\left(W_{r}\right)=\mu\left(W_{s}\right)$; then the corresponding elements of the algebra $A$ have equal $\mu$-measure, so (from (iii) of the theorem of $\left[8\right.$, p. 328]) $W_{r} \times Z \sim_{T} W_{8} \times Z$. Thus there exist disjoint subsets $H_{i}$ of $C-N_{2}$ such that the sets $T^{i}\left(H_{i}\right)$ are disjoint $(-\infty<i<\infty), m\left(W_{r}+{ }_{2} \cup H_{i}\right)=0$, and $m\left(W_{s}+{ }_{2} \cup T^{i}\left(H_{i}\right)\right)=0$. Hence, except for a set $Z_{r s} \subset Z$ with $\nu\left(Z_{r s}\right)=0$, we have that the sets $H_{i} \cap(Y \times x)$ are measurable in $(Y \times x, \mu), \mu\left\{\left(W_{r} \times x\right)+{ }_{2} \cup\left(H_{i} \cap(Y \times x)\right)\right\}=0$, and $\mu\left\{\left(W_{s} \times x\right)\right.$ $\left.{ }_{+}{ }_{2} \cup T^{i}\left(H_{i} \cap(Y \times x)\right)\right\}=0$. Write $X=Z-\cup Z_{r s}, N_{3}=N_{2} \cup\left(Y \times \cup Z_{r s}\right)$; then $N_{3}$ is null and invariant, and we have that (1) holds for each $x \in X$, provided that $Q_{1}$ and $Q_{2}$ are rational intervals. It follows that (1) holds (for $x \in X$ ). whenever $Q_{1}, Q_{2}$ are open sets, and thence $[7,4.11]$ provided only that $Q_{1}, Q_{2}$ are $G_{8}$ sets of finite measure; and from this (1) follows at once without restriction $\left({ }^{24}\right)$.

Restoring the suffix $n$, we define $\Omega_{n}=C_{n}-N_{3}$, and have established statements (i) and (ii) of Theorem 6, except that the product $Y_{n} \times X_{n}$ has a null set $N_{2}$ removed from it, where $\mu\left\{\left(Y_{n} \times x\right) \cap N_{2}\right\}=0$ for each $x \in X$. But it is

(24) Alternatively, the appeal to [7] can be replaced by a direct argument along the lines of the proof of 5.2(2) above. 
easy to construct an isometry of $\left(Y_{n} \times X_{n}\right)-N_{2}$, which is the identity except on an invariant null set, onto $Y_{n} \times X_{n}$, in such a way that $\left(Y_{n} \times x\right)-N_{2}$ is mapped onto $Y_{n} \times x$ isometrically; and (i) and (ii) are completely established when $Y_{n}$ is an interval.

Now (again dropping the suffix $n$ temporarily) suppose $(Y, \mu)$ is atomic, generated by $k$ atoms $q_{1}, \cdots, q_{k}$ of measure 1 ; and suppose $k$ is finite. By arguments similar to the preceding, we may suppose $C=Y \times Z$, and that each $Y \times x, x \in Z$, is invariant. We assert that, except for a set $Z_{1} \subset Z$ with $\nu\left(Z_{1}\right)=0$,

(2) the $k$ points $T^{i}\left(q_{1} \times x\right), 1 \leqq i \leqq k$, are distinct.

For otherwise there is a set $S \subset Z$, with $\nu(S)>0$, and a $q_{i}$, such that the invariant set $U T^{h}\left(q_{1} \times S\right)$ is disjoint from $q_{i} \times Z$. But (from the theorem of $[8$, p. 328]) every invariant subset of $C$ is (modulo null sets) of the form $Y \times S^{\prime}$, $S^{\prime} \subset Z$, giving a contradiction.

Write $X=Z-Z_{1}$, and consider the $k$ disjoint sets $T^{\boldsymbol{i}}\left(q_{1} \times X\right)$. Their union, say $\Omega$, is invariant, and $\Omega \subset C, m(C-\Omega)=0$. Further, the mapping $T^{h}\left(q_{1} \times X\right)$ $\rightarrow\left(p_{h} \times x\right)$ exhibits $\Omega\left(=\Omega_{n}\right)$ as a direct product in accordance with Theorem 6 .

The case $k=\infty$ is treated similarly.

All that remains to be proved is property (iii). From $[8,6.6]$, we have $Q_{1} \sim_{T} Q_{2}$ if and only if, for each $n, \Omega_{n} \cap Q_{1} \sim_{T} \Omega_{n} \cap Q_{2}$. But, from the construction in $[8,20.3],\left(\Omega_{n}, \sim_{T}\right)$ and $\left(Y_{n}, \mu_{n}\right) \times X_{n}$ are two realizations of the same abstract product. In $\left(Y_{n}, \mu_{n}\right) \times X_{n}$, the abstract measure of a set $Q$ is the function whose value, for almost every $x \in X_{n}$, is $\mu_{n}\left(Q \cap\left(Y_{n} \times x\right)\right)$; and the proof is complete.

7.3. For a measurable flow, we assume for simplicity that the total measure of $\Omega$ is finite, and have $\left({ }^{25}\right)$ :

THEOREM 7. If $T_{t}$ is a measurable flow on a normal measure space $(\Omega, m)$ $(m(\Omega)<\infty)$, then there exist disjoint invariant measurable subsets $\Omega_{1}, \Omega_{2}$, with $m\left(\Omega-\Omega_{1}-\Omega_{2}\right)=0$, such that

(i) on $\Omega_{1}, T_{t}$ is the identity,

(ii) on $\Omega_{2}$, the flow $T_{t}$ is isomorphic to a measurable flow $T_{t}^{\prime}$ on $(I, \lambda)$ $X(X, \mu)$, where $(I, \lambda)$ is the unit interval and $(X, \mu)$ is a $\sigma$-normal measure space (of finite measure), in such a way that, for each $x \in X, I \times x$ is invariant under $T_{t}^{\prime}$, and $T_{t}^{\prime}$ is an ergodic measurable flow on $(I \times x, \lambda)$.

(iii) Two measurable sets $Q_{1}, Q_{2}$, in $\Omega$ are equivalent under $T_{t}$ (in the sense of $\left[8\right.$, p. 328]) if and only if $m\left(\Omega_{1} \cap\left(Q_{1}+{ }_{2} Q_{2}\right)\right)=0$ and, for almost all $x \in X$, $\lambda\left((I \times x) \cap Q_{1}\right)=\lambda\left((I \times x) \cap Q_{2}\right)$.

This can be proved by observing that [1, Theorem 3$]$ gives a (direct sum) decomposition having some of these properties, and $[8$, p. 328] gives a decomposition having the others; and it is not hard to show (cf. proof of Theorem 5, 6.5 above) that these decompositions are essentially the same.

(25) Extending [1, Theorem 3]; compare also [10, p. 617]. See [2] for terminology. 
A similar theorem and proof can be given for "generalized flows" in the sense of von Neumann [10].

Finally, we remark that an alternative, and possibly more useful, "product-like" decomposition of a flow into ergodic parts can be obtained by combining the representation [2] of the flow as built under a function with Theorem 6 to decompose the transformation of the base-space into ergodic parts.

\section{BIBLIOGRAPHY}

1. W. Ambrose, P. R. Halmos, and S. Kakutani, The decomposition of measures II, Duke Math. J. vol. 9 (1942) pp. 43-47.

2. W. Ambrose and S. Kakutani, Structure and continuity of measuruble flows, Duke Math. J. vol. 9 (1942) pp. 25-42.

3. J. Dieudonné, Sur le théorème de Lebesgue-Nikodým III, Annales de l'Université de Grenoble vol. 23 (1948) pp. 25-53.

4. P. R. Halmos, The decomposition of measures, Duke Math. J. vol. 8 (1941) pp. 386-392. 42.

5. - On a theorem of Dieudonné, Proc. Nat. Acad. Sci. U.S.A. vol. 35 (1949) pp. 38-

6. P. R. Halmos and J. von Neumann, Operator methods in classical mechanics II, Ann. of Math. vol. 43 (1942) pp. 332-350.

7. D. Maharam, On measure in abstract sets, Trans. Amer. Math. Soc. vol. 51 (1942) pp. $413-433$.

8. - The representation of abstract measure functions, Trans. Amer. Math. Soc. vol. 65 (1949) pp. 279-330.

9. O. M. Nikodým, Tribus de Boole et fonctions mesurables, C. R. Acad. Sci. Paris vol. 228 (1949) pp. 150-151.

10. J. von Neumann, Zur operatorenmethode in der klassischen Mechanik, Ann. of Math. vol. 33 (1932) pp. 587-642.

11. S. Saks, Theory of the integral, Warsaw, 1937.

MANChESTER, ENGLAND. 